\title{
Regime Transitions of Steady and Time-Dependent Hadley Circulations: Comparison of Axisymmetric and Eddy-Permitting Simulations
}

\author{
SIMONA BORDONI* \\ National Center for the Atmospheric Research, ${ }^{+}$Boulder, Colorado \\ TAPIO SCHNEIDER \\ California Institute of Technology, Pasadena, California
}

(Manuscript received 24 August 2009, in final form 25 November 2009)

\begin{abstract}
Steady-state and time-dependent Hadley circulations are investigated with an idealized dry GCM, in which thermal forcing is represented as relaxation of temperatures toward a radiative-equilibrium state. The latitude $\phi_{0}$ of maximum radiative-equilibrium temperature is progressively displaced off the equator or varied in time to study how the Hadley circulation responds to seasonally varying forcing; axisymmetric simulations are compared with eddy-permitting simulations. In axisymmetric steady-state simulations, the Hadley circulations for all $\phi_{0}$ approach the nearly inviscid, angular-momentum-conserving limit, despite the presence of finite vertical diffusion of momentum and dry static energy. In contrast, in corresponding eddy-permitting simulations, the Hadley circulations undergo a regime transition as $\phi_{0}$ is increased, from an equinox regime ( small $\phi_{0}$ ) in which eddy momentum fluxes strongly influence both Hadley cells to a solstice regime (large $\phi_{0}$ ) in which the cross-equatorial winter Hadley cell more closely approaches the angular-momentum-conserving limit. In axisymmetric time-dependent simulations, the Hadley cells undergo transitions between a linear equinox regime and a nonlinear, nearly angular-momentum-conserving solstice regime. Unlike in the eddypermitting simulations, time tendencies of the zonal wind play a role in the dynamics of the transitions in the axisymmetric simulation. Nonetheless, the axisymmetric transitions are similar to those in the eddypermitting simulations in that the role of the nonlinear mean momentum flux divergence in the zonal momentum budget shifts from marginal in the equinox regime to dominant in the solstice regime. As in the eddy-permitting simulations, a mean-flow feedback-involving the upper-level zonal winds, the lower-level temperature gradient, and the poleward boundary of the cross-equatorial Hadley cell-makes it possible for the circulation fields to change at the transition more rapidly than can be explained by the steady-state response to the thermal forcing. However, the regime transitions in the axisymmetric simulations are less sharp than those in the eddy-permitting simulations because eddy-mean flow feedbacks in the eddy-permitting simulations additionally sharpen the transitions.
\end{abstract}

\section{Introduction}

Monsoons are generally viewed as regionally concentrated, thermally direct overturning circulations in the latitude-height plane, with ascending motion in the sum-

\footnotetext{
* Current affiliation: California Institute of Technology, Pasadena, California.

+ The National Center for Atmospheric Research is sponsored by the National Science Foundation.
}

Corresponding author address: Simona Bordoni, California Institute of Technology, Pasadena, CA 91125.

E-mail: bordoni@gps.caltech.edu mer hemisphere subtropics and descending motion in the winter hemisphere (Newell et al. 1972; Gadgil 2003; Bordoni and Schneider 2008). These monsoonal circulations dominate the solstitial zonally averaged Hadley circulation, which is characterized by a strong and broad cross-equatorial winter cell and a very weak and narrow summer cell. Most theories of the dynamics of these circulations have been developed in the context of axisymmetric models of the Hadley circulation in which the upper branches of the circulation are assumed to be nearly inviscid and angular-momentum-conserving (e.g., Schneider 1977; Held and Hou 1980; Lindzen and Hou 1988; Satoh 1994; Caballero et al. 2008). For instance, Plumb and Hou (1992) showed that axisymmetric 
circulations driven by a localized off-equatorial thermal forcing undergo transitions from a linear, viscous regime to a nonlinear, angular-momentum-conserving regime beyond a threshold forcing value; they suggested that this threshold behavior may account for the rapid onset of monsoons.

The nonlinear axisymmetric theory of Plumb and Hou (1992) has been extended in several studies to account for the influences of moist convection (Emanuel 1995; Zheng 1998), of a subtropical continent (Privé and Plumb 2007a,b), and of moisture-dynamics feedbacks such as wind-induced surface heat exchange (Boos and Emanuel 2008a,b). All of these studies, however, have postulated the existence of a localized subtropical heating (either provided by imposed surface temperature anomalies or a subtropical continent) as necessary for monsoon development and have neglected the interaction between large-scale eddies and tropical circulations.

But, large-scale eddies of midlatitude origin may in fact play an important role in the dynamics of Hadley and monsoonal circulations. Through idealized GCM experiments, Walker and Schneider (2006) found that over a wide range of climates, including earthlike climates, the strength of a Hadley cell driven by hemispherically symmetric thermal forcing is strongly influenced by eddy momentum fluxes of extratropical origin, so the scalings that nearly inviscid axisymmetric theory gives for the extent and strength do not apply. However, as the thermal forcing is made progressively more asymmetric about the equator, the cross-equatorial Hadley cell undergoes a transition from a regime in which its strength is strongly tied to eddy momentum fluxes to a regime in which the influence of the eddies is weaker and the angularmomentum-conserving limit is more closely approached. A similar transition also occurs in a simple single-layer axisymmetric model in which eddy momentum fluxes are parameterized (Sobel and Schneider 2009). Schneider and Bordoni (2008, hereafter SB08) showed that, over the course of seasonal cycles simulated with an idealized GCM without a hydrological cycle and with a uniform lower boundary, the Hadley cell undergoes rapid transitions between the eddy-dominated regime around the equinoxes and a more closely angular-momentumconserving regime around the solstices. SB08 discuss how feedbacks among large-scale eddy fluxes, upperlevel winds, and the tropical overturning circulation mediate these transitions and render them rapid. Bordoni and Schneider (2008) used reanalysis data to show that the onset of the Asian monsoon marks a transition between the two circulation regimes; simulations using an aquaplanet GCM with an active hydrological cycle demonstrated that rapid, eddy-mediated monsoon transitions can occur in the absence of surface inhomogeneities, provided the lower boundary has low enough thermal inertia.

The importance of large-scale eddies and of their interaction with the tropical circulation for the existence and rapidity of monsoon transitions can be evaluated by comparing eddy-permitting simulations with axisymmetric (eddy-suppressing) simulations. Here we use the same idealized GCM as in SB08 in an axisymmetric configuration to simulate steady states and seasonal cycles of axisymmetric Hadley cells and to explore if and to what extent regime transitions similar to those in the eddypermitting simulations still occur in the absence of eddies. Our approach is similar to that used by Fang and Tung (1999), who have also studied axisymmetric, timedependent Hadley circulations driven by seasonally varying thermal forcing. But our radiative heating formulation and the parameter space that we explore are different, as is our emphasis on a comparison with the regime transitions occurring in eddy-permitting simulations.

After a brief description of the model in section 2, we present the steady-state simulations in section 3 . Section 4 discusses results from time-dependent simulations of seasonal cycles. Conclusions follow in section 5 .

\section{Idealized GCM}

The idealized GCM is the same hydrostatic primitiveequation model as in SB08, where more details can be found. The model is a spectral-transform model, run in axisymmetric configuration (truncated at zonal wavenumber zero) with T42 horizontal resolution and 30 unequally spaced sigma levels in the vertical. Radiative forcing is provided by Newtonian relaxation toward a radiative-equilibrium state of a semigray atmosphere, which is axisymmetric and statically unstable in the lower troposphere. The radiative-equilibrium surface temperature varies with latitude as

$$
T_{s}^{e}=\max \left\{200 \mathrm{~K}, 350 \mathrm{~K}-\Delta_{h}\left[\sin ^{2} \phi-2 \sin \phi_{0}(t) \sin \phi\right]\right\},
$$

where $\phi_{0}$ is the latitude at which $T_{s}^{e}$ is maximal, and $\Delta_{h}=$ $112.5 \mathrm{~K}$ is the pole-to-equator temperature difference for $\phi_{0}=0$. In the steady-state simulations, $\phi_{0}$ is a fixed parameter; in the time-dependent simulations, it varies with time according to

$$
\phi_{0}(t)=23.5^{\circ} \sin (2 \pi t / 365 \text { day })
$$

This thermal forcing fundamentally differs from that used in Plumb and Hou (1992) in that it is not localized in the subtropics and in that the radiative-equilibrium 
temperature has nonzero curvature and (for $\phi_{0} \neq 0$ ) a nonzero gradient at the equator. This implies that a meridional circulation is to be expected for all values of $\phi_{0}$ (Plumb and Hou 1992). It also differs from that used in Fang and Tung (1999) in that it features larger seasonal excursions of the $T_{s}^{e}$ maximum away from the equator.

Turbulent dissipation in a planetary boundary layer of fixed height $(2.5 \mathrm{~km})$ is represented as vertical diffusion of momentum and dry static energy. Above the boundary layer, vertical diffusion of momentum and dry static energy with Prandtl number one and constant diffusivity $5 \mathrm{~m}^{2} \mathrm{~s}^{-1}$ needs to be included in the axisymmetric simulations to achieve steady states. This diffusivity is comparable to that used in previous studies of axisymmetric Hadley circulations (Lindzen and Hou 1988; Fang and Tung 1999; Walker and Schneider 2005) and allows the nearly inviscid limit to be approached, even for small $\phi_{0}$ values. However, it is not quite large enough to suppress symmetric instabilities entirely for larger $\phi_{0}$ values, which leads to "noise" in some of the plots.

The model does not include a hydrological cycle. However, the stabilizing effect of latent heat release is mimicked by a quasi-equilibrium convection scheme, which relaxes temperatures in an atmospheric column to a profile with lapse rate $0.7 \Gamma_{d}=6.8 \mathrm{~K} \mathrm{~km}^{-1}$, with dry adiabatic lapse rate $\Gamma_{d}$, if an atmospheric column is less stable than a column with lapse rate $0.7 \Gamma_{d}$. The convective relaxation time in the axisymmetric simulations is 1 day, six times larger than the value $(4 \mathrm{~h})$ used in the eddypermitting simulations in SB08. Such a large convective time scale allows the circulations to reach steady states (or nearly steady states) with smaller vertical diffusivities. Axisymmetric simulations with a convective time scale of $4 \mathrm{~h}$ and vertical diffusivities of $10 \mathrm{~m}^{2} \mathrm{~s}^{-1}$ do not differ substantially from the ones reported here.

Steady-state simulations were conducted with fixed values of $\phi_{0}$ ranging from $0^{\circ}$ (vernal equinox) to $23.5^{\circ} \mathrm{N}$ (boreal summer solstice). For comparison with the results from these steady-state axisymmetric simulations, in section 3 we also show results from the statistically steady states of the eddy-permitting simulations in SB08. The averages shown are surface-pressure-weighted sigmacoordinate averages over longitude and time (over 100 simulated days) in the axisymmetric and eddy-permitting simulations. The time-dependent simulation of seasonal cycles was started from the equinox steady state $\left(\phi_{0}=0^{\circ}\right)$ and was run for five years. The results shown in section 4 are from the equilibrated response, which is reached three years into the simulation. Our discussion mostly focuses on the comparison of the axisymmetric time-dependent simulations with the eddy-permitting simulations in SB08

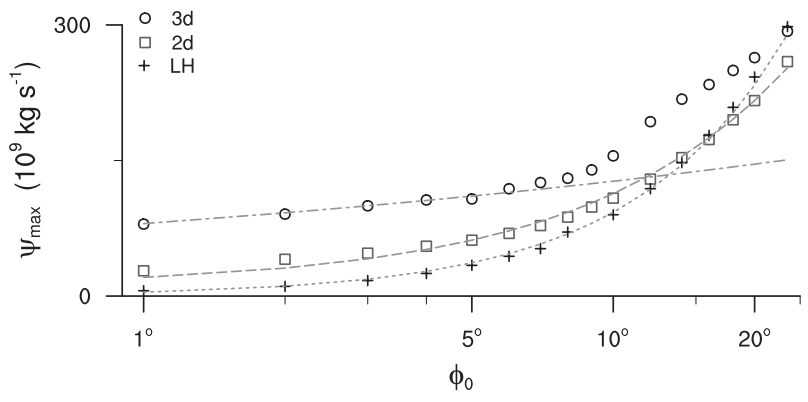

FIG. 1. Strength $\Psi_{\max }$ of the cross-equatorial Hadley cell as a function of $\phi_{0}$ in axisymmetric simulations of steady states (open squares). For comparison, corresponding Hadley cell strengths from the eddy-permitting simulations in SB08 and from nearly inviscid axisymmetric calculations analogous to those of Lindzen and Hou (1988) are shown as open dots and crosses, respectively. The gray dashed, dotted, and dashed-dotted lines indicate linear, $\phi_{0}^{4 / 3}$, and $\phi_{0}^{1 / 5}$ power laws. In the simulations, the strength is the maximum absolute value of the streamfunction of the mean meridional circulation above the level $\sigma=0.85$. The scale of the $x$ axis is logarithmic.

(control). We also discuss how the longer convective time scale and the nonzero vertical diffusivities used in the axisymmetric simulations impact our results, by comparing the control eddy-permitting simulation with eddypermitting simulations in which the longer convective time scale and the vertical diffusivities are separately or simultaneously introduced.

\section{Steady states}

Figure 1 shows the strength of the cross-equatorial Hadley cell in the axisymmetric steady-state simulations for different values of the latitude $\phi_{0}$ of maximum radiative-equilibrium surface temperature, together with the corresponding values from the eddy-permitting simulations in SB08. In the eddy-permitting simulations, the scaling of the cross-equatorial Hadley cell strength as a function of $\phi_{0}$ is in two different regimes: a weaker dependence on $\phi_{0}$ for $\phi_{0} \lesssim 9^{\circ}$ (roughly $\phi_{0}^{1 / 5}$ ) and a stronger dependence for $\phi_{0}$ for $\phi_{0} \gtrsim 9^{\circ}$ (roughly $\phi_{0}^{3 / 4}$ ). In contrast, in the axisymmetric simulations, the crossequatorial Hadley cell strength increases almost linearly with $\phi_{0}$ throughout the parameter space. For the largest $\phi_{0}$ values, the Hadley cell strengths in the eddypermitting and axisymmetric simulations converge. In Fig. 1, we also show the strength of the cross-equatorial Hadley cell from numerical calculations analogous to those of Lindzen and Hou (1988) but with the radiativeconvective equilibrium state of our simulations. Similarly to what is seen in the axisymmetric simulations, the nearly inviscid axisymmetric theory for our simulations does not exhibit a transition in scaling regimes at $\phi_{0} \sim 9^{\circ}$, but it predicts a somewhat stronger power-law dependence of 


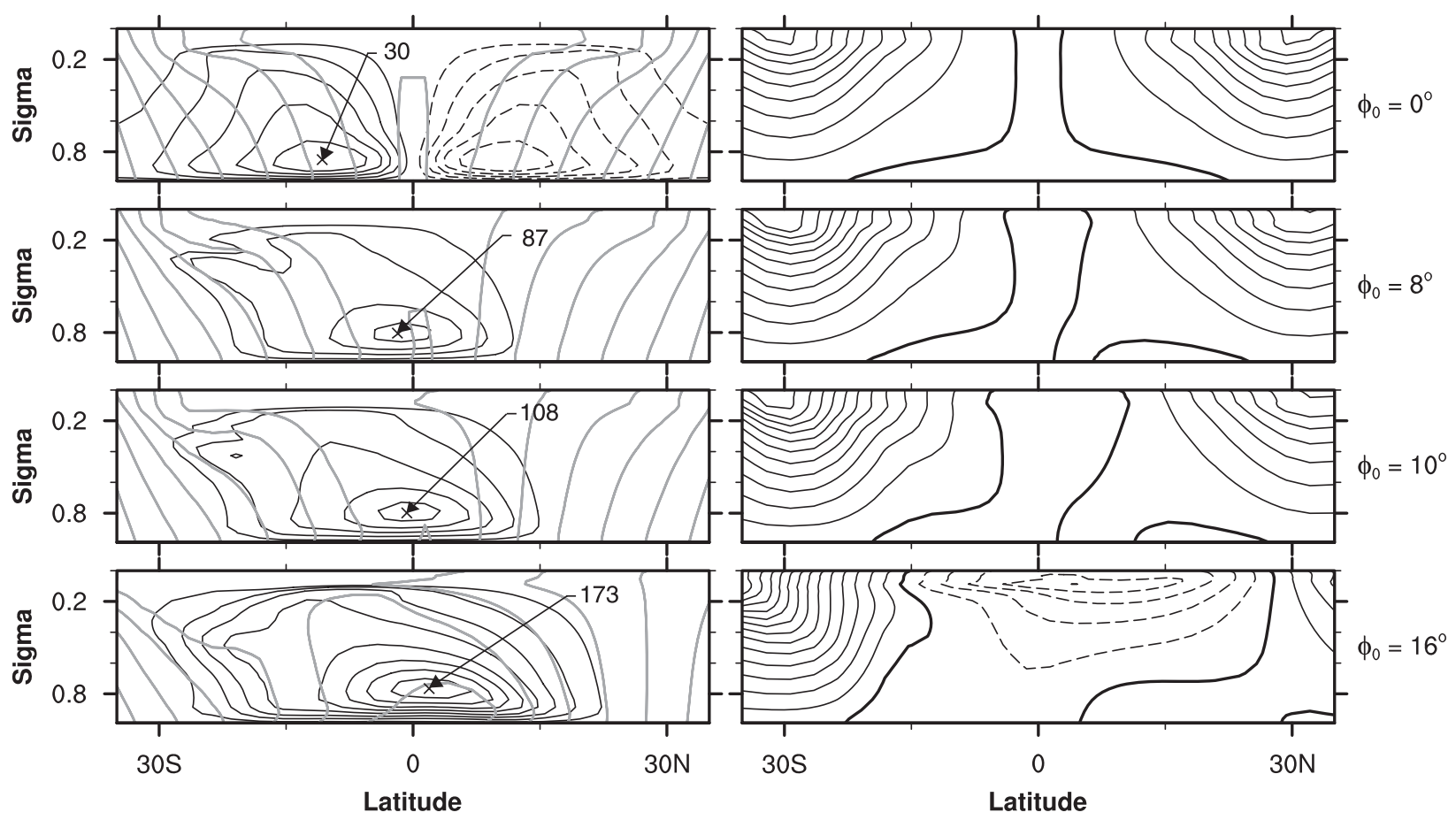

FIG. 2. Steady-state circulations. Rows correspond to the latitude $\phi_{0}$ of maximum radiative-equilibrium surface temperature indicated on the right. In the left column, black contours indicate streamfunction of meridional circulations (contour interval $5 \times 10^{9}$ in the first row and $20 \times 10^{9} \mathrm{~kg} \mathrm{~s}^{-1}$ in rows 2-4), with solid contours for positive values (counterclockwise rotation) and dashed contours for negative values (clockwise rotation). Gray contours indicate absolute angular momentum per unit mass $\bar{m}=(\Omega a \cos \phi+\bar{u}) a \cos \phi$, with $\operatorname{contours~at}$ $\Omega a^{2} \cos ^{2} \phi_{i}\left(\phi_{i}=0^{\circ}, \pm 5^{\circ}, \pm 10^{\circ}, \ldots\right)$, to give angular momentum contours at $5^{\circ}$ latitude intervals in the absence of zonal flow. Arrows identify streamfunction maxima above $\sigma=0.85$, with maximum values given in units of $10^{9} \mathrm{~kg} \mathrm{~s}^{-1}$. In the right column, black contours indicate zonal winds (contour interval $10 \mathrm{~m} \mathrm{~s}^{-1}$; negative contour dashed and zero contour thick).

the circulation strength on $\phi_{0}$ (roughly $\left.\phi_{0}^{4 / 3}\right)$. The axisymmetric cross-equatorial circulations do not exhibit a transition in scaling regimes in the parameter space because they tend to approach the angular-momentumconserving limit for all values of $\phi_{0}$, despite the finite vertical diffusion of momentum and dry static energy.

The nearly angular-momentum-conserving nature of the axisymmetric circulations is suggested in Fig. 2, which shows streamlines of the mean meridional circulation, angular momentum contours, and zonal winds for four different values of $\phi_{0}$. In the angular-momentumconserving limit, streamlines and angular momentum contours coincide, and it is apparent that this is approximately the case throughout the upper branches of the circulations. For $\phi_{0}=0^{\circ}$ (row 1 in Fig. 2), the tropical circulation has a northern and a southern cell symmetric about the equator. Diffusive vertical mixing of momentum and dry static energy is clearly nonnegligible near the subtropical edges of the two cells, where strong shear zones in zonal winds form (cf. Held and Hou 1980); it leads to streamlines crossing angular momentum contours in the descending branch of the cells. However, in the deep tropics, angular momentum contours are strongly displaced from the vertical, indicating significant nonlinear advection and homogenization of angular momentum by the mean meridional circulation in the cells' upper branches. In this region, from the equator up to about $10^{\circ}$ latitude, the upper-level zonal wind approaches its angular-momentum-conserving limit, not deviating by more than $15 \%$ from it poleward of $4^{\circ}$ latitude (Fig. 3). The local Rossby number Ro $=-\bar{\zeta} / f$, with relative vorticity $\zeta$ and planetary vorticity $f$, is a nondimensional measure of the proximity of the circulation to the angular-momentum-conserving limit (e.g., Schneider 2006) and reaches Ro $\sim 0.7-0.8$ in the upper branches of the cells near the latitudes of streamfunction extrema.

As $\phi_{0}$ is progressively displaced off the equator, the southern (cross-equatorial) cell intensifies and broadens, while the northern cell weakens and narrows (rows 2-4 in Fig. 2). Unlike in the eddy-resolving simulations (see Fig. 3 in SB08), however, the strength of the southern cell does not intensify rapidly, and the streamfunction maximum does not move across the equator into the summer hemisphere rapidly, at a critical $\phi_{0}$ value. The streamfunction maximum moves gradually from $\sim 10^{\circ}$ in 


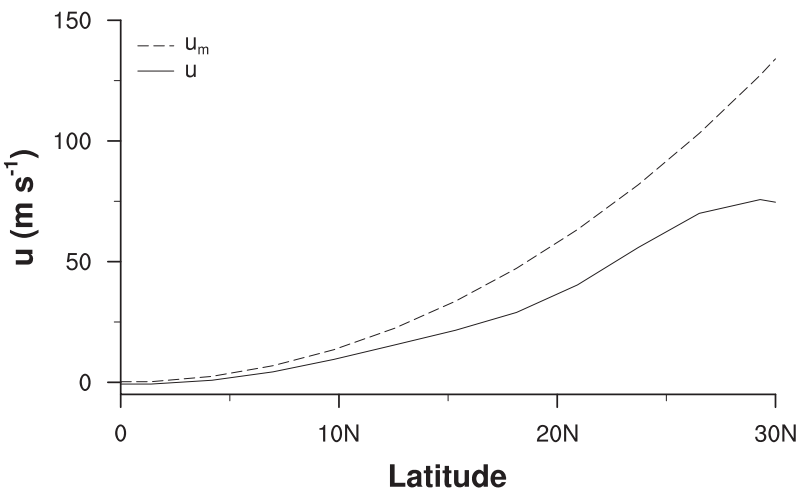

FIG. 3. Upper-level $(\sigma=0.2)$ zonal winds in the equinoctial Hadley cells from the steady-state axisymmetric simulation with $\phi_{0}=0^{\circ}$ (solid) and angular-momentum-conserving winds, $u_{m}=$ $\Omega a \sin ^{2} \phi / \cos \phi$ (dashed).

the Southern Hemisphere at $\phi_{0}=0^{\circ}$ to near the equator at $\phi_{0}=16^{\circ}$. It is always located in a region where, at upper levels, streamlines and angular momentum contours tend to coincide. Zonal winds are within $10 \%$ of the angular momentum-conserving winds over much of the upper branch of the cross-equatorial Hadley cell. Deviations from the angular-momentum-conserving limit are significant only near the poleward edge of the cell in its descending branch, where vertical diffusion makes it possible for the streamlines to cross angular momentum contours. As the southern cell acquires a crossequatorial component, low-level westerly flow develops north of the equator up to the poleward boundary of the cross-equatorial cell, as required by the approximate balance in the boundary layer between the Coriolis force on the meridional near-surface flow and the drag on the near-surface zonal wind (SB08).

The relative role of diffusion and nonlinear advection of momentum in the Hadley cells can be evaluated more quantitatively by decomposing the total streamfunction $\Psi$ into a component $\Psi_{m}$ associated with the nonlinear mean momentum flux divergence $\mathcal{M}$ and a residual component $\Psi_{r}$, which above the boundary is mainly viscously driven (in the boundary layer, the residual streamfunction has a component associated with the turbulent drag on the zonal wind). The zonal momentum budget in steady state can be written as

$$
f \bar{v}=\mathcal{M}+\mathcal{R},
$$

where $\mathcal{R}$ denotes the viscous stresses in the interior and turbulent drag in the boundary layer. This can be integrated in height to yield

$$
\Psi=\Psi_{m}+\Psi_{r},
$$

where

$$
\Psi_{m}(\phi, p)=-\frac{2 \pi a \cos \phi}{f g} \int_{0}^{p} \mathcal{M} d p^{\prime}
$$

is the component of the mean meridional circulation associated with nonlinear mean momentum fluxes. ${ }^{1}$ Figure 4 shows the streamfunction components $\Psi_{m}$ and $\Psi_{r}$ for the same four values of $\phi_{0}$ as in Fig. 2. It is evident that the nonlinear component dominates in the equinoctial and cross-equatorial Hadley cell for all $\phi_{0}$ in the regions around the global streamfunction maximum. The viscous component dominates only locally in the descending branch of the Hadley cell.

The behavior of $\Psi_{m}$ and $\Psi_{r}$ as $\phi_{0}$ increases is made more explicit in Fig. 5a, which shows the two streamfunction components at the center of the cross-equatorial Hadley cell (i.e., at the latitude and level of the streamfunction extremum). For all $\phi_{0}$, the largest contribution to the streamfunction is provided by the nonlinear component, with a roughly linear dependence of $\Psi_{m}$ on $\phi_{0}$ and no transition in scaling regime. This demonstrates that in all axisymmetric simulations, the right-hand side of the zonal momentum budget (3) near the center of the crossequatorial cell is dominated by the nonlinear mean momentum flux divergence. This is in contrast to the eddy-permitting simulations (Fig. 5b) in which the transition in scaling regimes of the cross-equatorial Hadley cell at $\phi_{0} \approx 9^{\circ}$ marks a shift in the dominant balance in the zonal momentum budget (3), from eddy momentum fluxes dominating the right-hand side for $\phi_{0} \lesssim 9^{\circ}$ to the nonlinear mean momentum flux dominating for $\phi_{0} \geqslant 9^{\circ}$.

These results suggest that the equinoctial and crossequatorial Hadley cell in the axisymmetric simulations responds directly to variations in the thermal forcing for all $\phi_{0}$ values. In contrast, in the eddy-permitting simulations in SB08, as $\phi_{0}$ is displaced off the equator, the cross-equatorial Hadley cell undergoes a transition from an eddy-dominated regime, in which the cell strength responds only indirectly to changes in the thermal forcing, to a nearly angular-momentum-conserving regime, in which the cell strength responds more directly to

\footnotetext{
${ }^{1}$ As in SB08, for simplicity, we use pressure coordinates in equations throughout the paper. The simulation results are based on averages in the model's vertical sigma coordinate, for which means along isobars are replaced by surface-pressure-weighted averages along sigma surfaces.

${ }^{2}$ In the eddy-permitting simulations, above the planetary boundary layer, the term $\mathcal{R}$ in the zonal momentum budget [(3)] is associated with the eddy momentum flux divergence. For details, see SB08.
} 


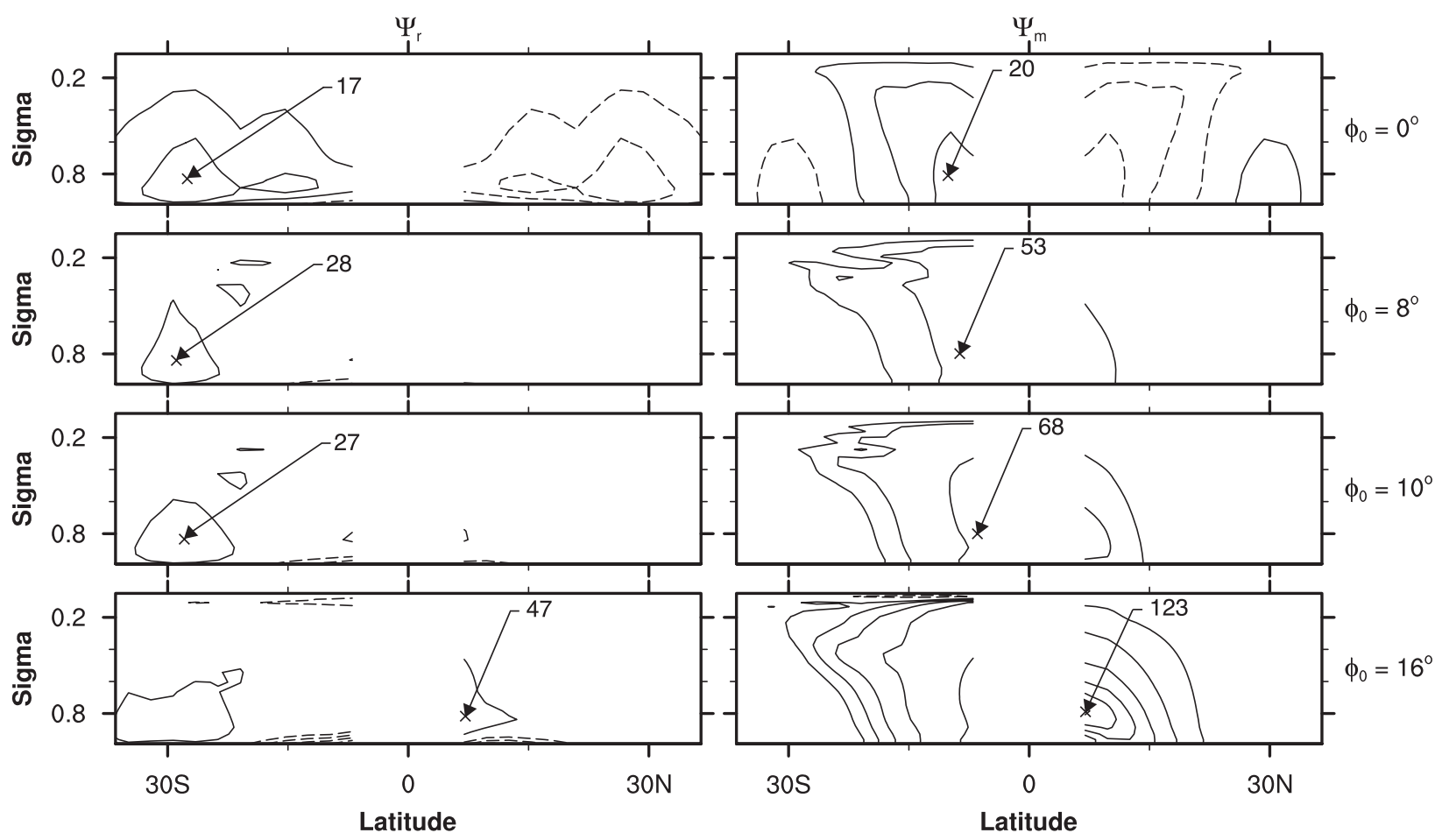

FIG. 4. Streamfunction components associated with (left) viscous stresses and (right) nonlinear mean momentum flux divergence. Rows correspond to the same four latitudes $\phi_{0}$ of maximum radiative-equilibrium temperature as in Fig. 2. The streamfunction components are calculated as $\Psi_{m}=-2 \pi a /(f g) \int_{0}^{\sigma} \operatorname{div}\left[\bar{p}_{s} \cos \phi\left(\bar{u}^{\sigma} \bar{v}^{\sigma}, \bar{u}^{\sigma} \bar{\sigma}^{\sigma}\right)\right] d \sigma^{\prime}$, where $\dot{\sigma}=D \sigma / D t$ and $\Psi_{r}=\Psi-\Psi_{m}$. Streamfunction components within $4.2^{\circ}$ of the equator, where they are poorly defined because the Coriolis parameter approaches zero, are not shown. Contours are as in Fig. 2 (left). Arrows identify streamfunction maxima above $\sigma=0.85$, with maximum values given in units of $10^{9} \mathrm{~kg} \mathrm{~s}^{-1}$.

changes in the thermal forcing (see discussion in Schneider 2006; Walker and Schneider 2006; SB08).

The lack of a transition in circulation regimes in the axisymmetric steady-state simulations is also reflected in smooth dependences of other circulation fields on $\phi_{0}$. In the eddy-permitting simulations, at the transition of the southern Hadley cell from the eddy-dominated regime to the nearly angular-momentum-conserving regime at $\phi_{0} \sim 9^{\circ}$, the streamfunction maximum jumps across the equator from the winter into the summer hemisphere. Figure $6 \mathrm{~d}$ shows this shift for the streamfunction maximum at the fixed lower-level $\sigma=0.85$, which is close to the level at which both the axisymmetric and eddy-permitting Hadley cells have their overall streamfunction maxima (see Fig. 2 here and Fig. 3 in SB08). Because by continuity the upward mass flux at any given level vanishes at the streamfunction extremum at that level, this shift is also accompanied by a jump in the position of the inner boundary of the region of upward mass flux to near the equator. At the transition, the strength of the lower-level upward mass flux increases markedly, from $\sim 9 \times 10^{4} \mathrm{~kg} \mathrm{~m}^{-1} \mathrm{~s}^{-1}$ at $\phi_{0}=8^{\circ}$ right before the transition to $\sim 24 \times 10^{4} \mathrm{~kg} \mathrm{~m}^{-1} \mathrm{~s}^{-1}$ at $\phi_{0}=16^{\circ}$ (Fig. 6b). None of these features are evident in the axisymmetric simulations in which the lower-level streamfunction maximum, and with it the inner boundary of the region of upward mass flux, move gradually from the winter hemisphere into the summer hemisphere (Fig. 6c). There is no rapid shift across the equator, and the maximum values of the lower-level upward mass flux increase gradually with $\phi_{0}$, for example, from $\sim 5 \times$ $10^{4} \mathrm{~kg} \mathrm{~m}^{-1} \mathrm{~s}^{-1}$ at $\phi_{0}=8^{\circ}$ to $\sim 10 \times 10^{4} \mathrm{~kg} \mathrm{~m}^{-1} \mathrm{~s}^{-1}$ at $\phi_{0}=16^{\circ}$ (Fig. 6a).

It is also of interest to explore how local circulation fields in the subtropics, which in the eddy-permitting simulations undergo rapid shifts at the regime transition, behave in the axisymmetric experiments. Figure 7 shows the lower-level upward mass flux and zonal wind at $13^{\circ} \mathrm{N}$. No rapid changes are seen in the axisymmetric simulations. In particular, the lower-level zonal wind remains westerly for all values of $\phi_{0}$, in contrast to the reversal seen in the eddy-permitting simulations and at the onset of the earth's large-scale monsoons (e.g., Webster 1987).

If the axisymmetric steady states described above were indeed achieved in time-dependent simulations with a seasonally varying thermal forcing, the results shown here would lead us to conclude that, in the absence of 
a

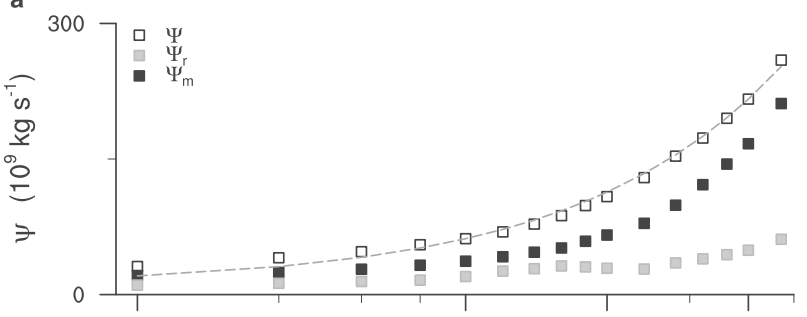

b

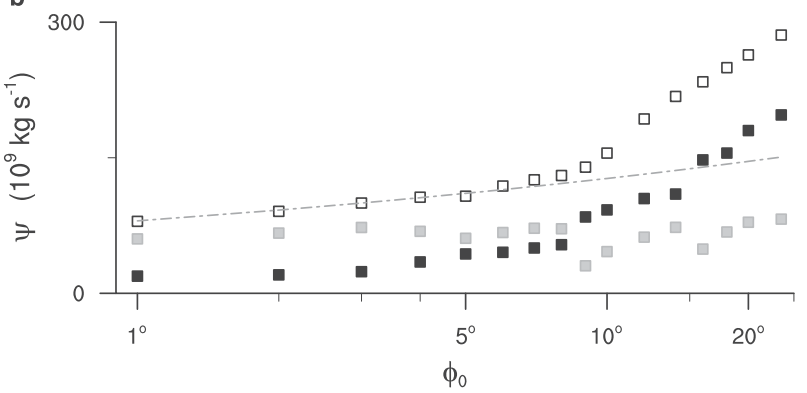

FIG. 5. Streamfunction components as a function of $\phi_{0}$ in (a) axisymmetric and (b) eddy-permitting simulations of steady states. Strength of the cross-equatorial Hadley cell $\Psi_{\max }$ (open squares) and $\Psi_{m}$ and $\Psi_{r}$ values (filled gray and black squares, respectively) at the center of the cell. The gray dashed and dashed-dotted lines indicate $\phi_{0}^{1}$ and $\phi_{0}^{1 / 5}$ power laws in (a) and (b) respectively, as in Fig. 1. In the eddy-permitting simulations, $\Psi_{r}$ is associated with the eddy-momentum fluxes and computed as $\Psi_{r}=$ $-2 \pi a /(f g) \int_{0}^{\sigma} \operatorname{div}\left[\bar{p}_{s} \cos \phi\left({\overline{u^{\prime} v^{\prime}}}^{\sigma},{\overline{u^{\prime} \dot{\sigma}^{\prime}}}^{\sigma}\right)\right] d \sigma^{\prime}$.

large-scale eddies, the tropical overturning circulation would not undergo regime transitions. However, as noted, for example, by Plumb and Hou (1992), Fang and Tung (1999), and Boos and Emanuel (2008a), the adjustment time for the axisymmetric circulations to achieve a steady state is longer than 100 days, that is, longer than the seasonal time scale. So there is no guarantee that steady-state circulations are relevant to the seasonal cycle of axisymmetric Hadley circulations. It is therefore of interest to study the time-dependent circulation corresponding to periodic $\phi_{0}$ variations (2) and to compare it with the steady circulations. This is done in the next section.

\section{Seasonal cycle}

Figure 8 shows the same quantities as in Fig. 2 but for four pentads in the course of the seasonal cycle in an axisymmetric simulation. At day 1-5 (first row in Fig. 8), immediately after the vernal equinox, the tropical circulation is characterized by the equinoctial pattern, with two cells with ascending branches located near the equator. Unlike in the corresponding steady state with $\phi_{0}=0^{\circ}$, however, the two cells deviate significantly from the angular-momentum-conserving regime. This is particularly evident throughout the southern cell where streamlines of the meridional circulation cross angular momentum contours, which are only slightly distorted from the vertical. The streamfunction maximum is located farther away from the equator than in the corresponding steady-state cell (at about $20^{\circ}$ into the Southern Hemisphere), in a region where local Rossby numbers in the upper branch do not exceed Ro $\sim 0.4$. The structure of the zonal winds is also different, with easterly flow spanning broader regions in the tropics, both at upper and lower levels. An analysis of the time- and zonal-mean zonal momentum budget reveals that in the upper branch of the southern cell, above the streamfunction maximum, the nonlinear mean momentum flux divergence associated with the mean meridional circulation is weak, and the mean meridional advection of planetary angular momentum —or the Coriolis force on the meridional flow-is primarily balanced by the time tendency of the zonal wind. The equinox cell, therefore, is in an approximately linear, transient regime in which the circulation has not reached equilibrium with the forcing and significantly deviates from angular-momentum-conserving flow.

At day 11-15 (Fig. 8, second row), the southern cell has strengthened and the northern cell has weakened. The cell is still in the approximately linear, transient regime in which the dominant momentum balance in the upper branch is between the Coriolis force on the meridional flow and the time tendency term. The circulation is unable to homogenize angular momentum: angular momentum contours are almost vertical throughout the cell. The streamfunction maximum of the southern cell has moved toward the equator and is now located at $\sim 10^{\circ} \mathrm{S}$. Local Rossby numbers in the upper branch above the streamfunction maximum remain relatively small, with maximum values Ro $\sim 0.4-0.5$.

By day 26-30 (Fig. 8, third row), the southern cell is undergoing a transition from the approximately linear, transient regime to a nonlinear, nearly angular-momentumconserving regime. In 15 days, the streamfunction maximum has moved to a location close to the equator. At upper levels, the zonal wind tendency term is small, and the dominant balance in the zonal momentum budget is between the Coriolis force on the meridional flow and the nonlinear angular momentum flux divergence. In the ascending and upper branch above the center of the cell, streamlines and angular momentum contours almost coincide. As the latitude $\phi_{0}$ of maximum radiativeequilibrium surface temperature is further displaced into the Northern Hemisphere, the southern cell keeps intensifying and broadening, with its maximum remaining fairly close to the equator and with an almost angularmomentum-conserving upper branch. Once in the nearly angular-momentum-conserving regime, the tropical 


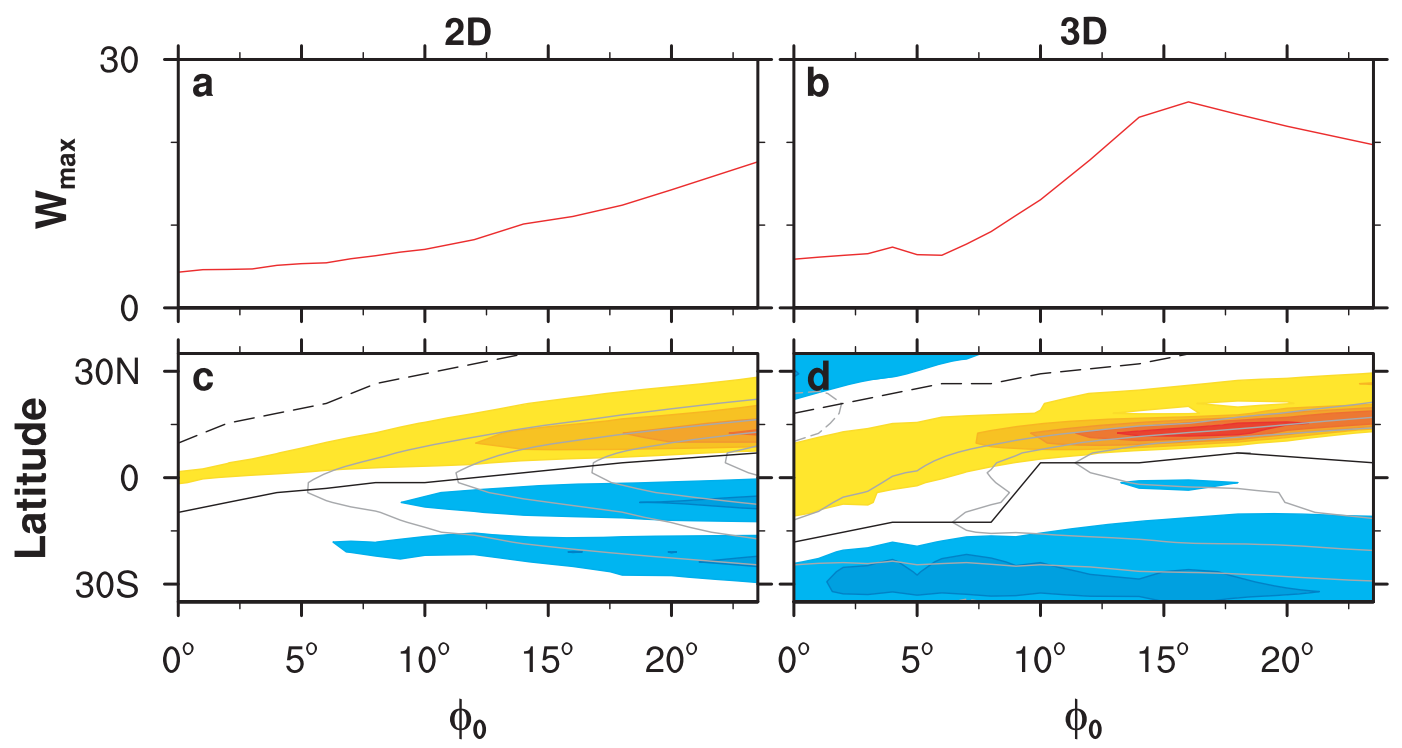

FIG. 6. Lower-level ( $\sigma=0.85)$ circulation fields as a function of $\phi_{0}$ in steady-state simulations. (top) Maximum values of upward mass flux $W=-\mu \bar{\sigma}^{\sigma}\left(10^{4} \mathrm{~kg} \mathrm{~m}^{-1} \mathrm{~s}^{-1}\right)$ in the southern Hadley cell for (a) axisymmetric and (b) eddypermitting simulations, with atmospheric mass per unit length in a meridional cross section, $\mu=2 \pi a \cos \phi \times \bar{p}_{s} / g$. (bottom) Upward mass flux $W$ (colors, contour interval $4 \times 10^{4} \mathrm{~kg} \mathrm{~m}^{-1} \mathrm{~s}^{-1}$ ), streamfunction of mean meridional circulation (gray contours; contour interval $60 \times 10^{9} \mathrm{~kg} \mathrm{~s}^{-1}$ ), and position of streamfunction maximum and minimum (solid and dashed black line) for (c) axisymmetric and (d) eddy-permitting simulations.

circulation and associated zonal wind patterns resemble closely those of the corresponding steady states. The circulation at summer solstice is shown in the bottom row of Fig. 8. As in the steady states, diffusive vertical mixing of momentum and dry static energy is nonnegligible only near the poleward edge of the cross-equatorial cell in its descending branch, making it possible for streamlines to cross angular momentum contours there.

Thus, the time-dependent axisymmetric Hadley cells undergo transitions from an approximately linear, transient regime to a nearly nonlinear, angular-momentumconserving regime. That the regime transitions of the time-dependent Hadley cells indeed mark a shift in the dominant balance in the zonal momentum budget can be seen in Fig. 9. The figure shows the seasonal evolution of the streamfunction components $\Psi_{m}$, which is associated with the nonlinear mean momentum flux divergence $\mathcal{M}$ as in the steady-state simulations, and $\Psi_{r}$, which is associated with viscous stresses and time tendencies of the zonal wind, with both $\Psi_{m}$ and $\Psi_{r}$ evaluated at the center of the southern Hadley cell. The role of the mean momentum flux divergence in the zonal momentum budget clearly shifts from marginal in the equinox regime to dominant in the solstice regime. In this respect, the transitions of the time-dependent axisymmetric Hadley circulations are similar to those in the eddypermitting simulations in SB08 in that they mark shifts in the dominant momentum balance from a linear to a nonlinear regime. However, the dynamics of the transitions in the axisymmetric and eddy-permitting simulations differ in that time tendencies of the zonal wind do not play a significant role in the eddy-permitting simulations but do in the axisymmetric simulations. The

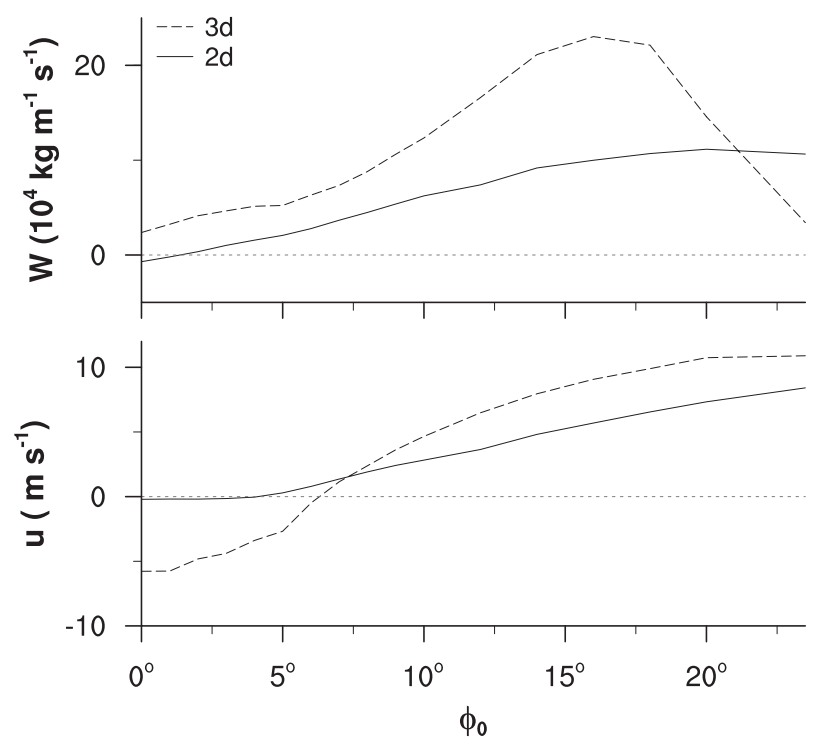

FIG. 7. Lower-level fields at $13^{\circ}$ latitude as a function of $\phi_{0}$ in steady-state simulations. (top) Upward mass flux $W$ and (bottom) zonal winds from axisymmetric (solid) and eddy-permitting (dashed) simulations. 

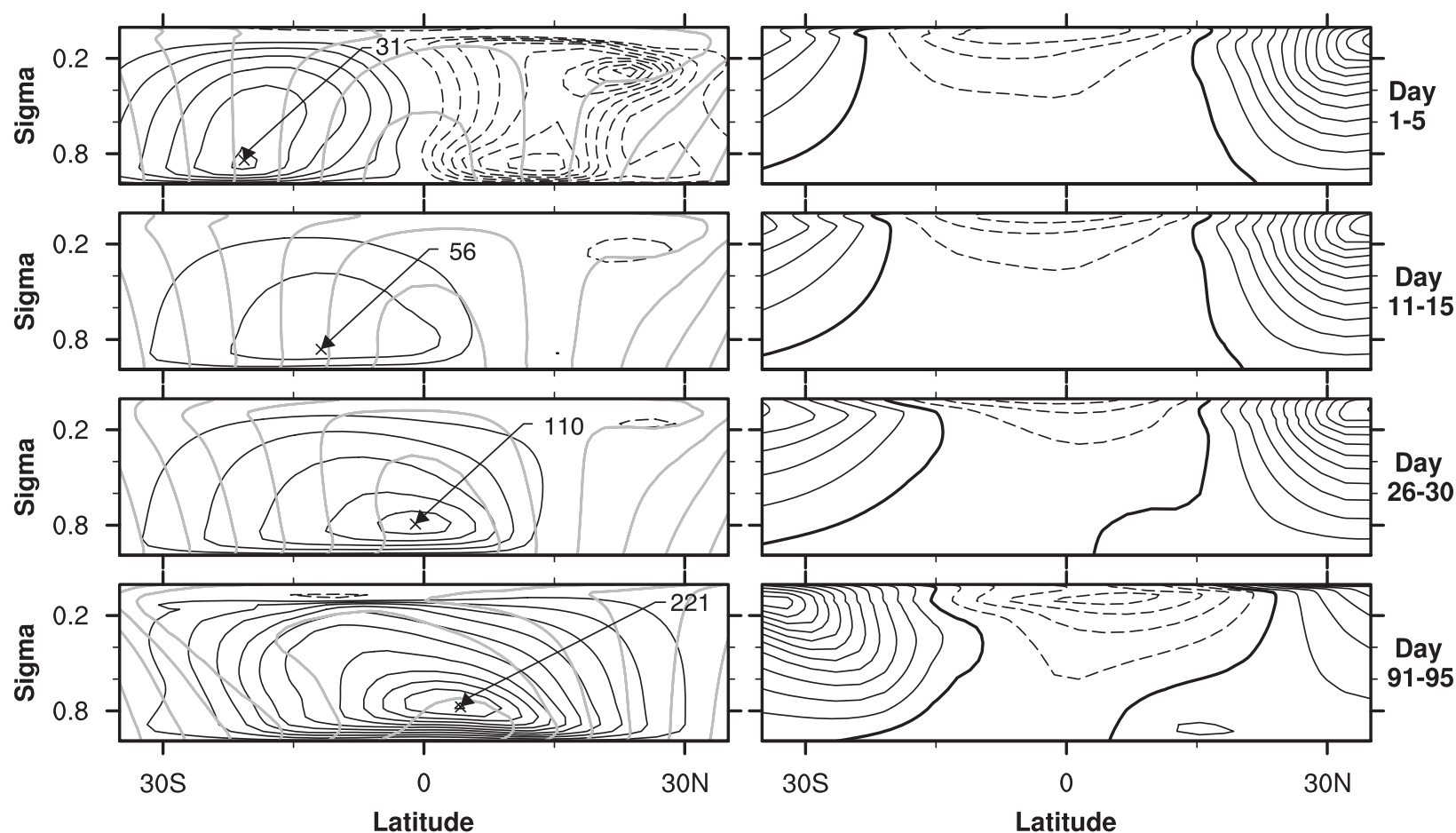

FIG. 8. Mean circulations at four pentads in the course of the seasonal cycle in an axisymmetric simulation. Rows correspond to the pentads indicated on the right (in days after vernal equinox). Quantities and contour intervals are as in Fig. 2.

greater importance of transience in the axisymmetric simulations is a viscous effect, as the viscosity influences the adjustment time scale of the circulations (because eddies respond more rapidly, the adjustment time scale in the eddy-permitting simulations is shorter). The transient nature of the equinox pattern in the seasonal cycle of axisymmetric Hadley circulations is also evident in the simulations of Fang and Tung (1999) in which a strictly equatorially symmetric circulation is never achieved. Fang and Tung concluded that the strength of time-dependent Hadley circulations is not as sensitive to the asymmetry of the thermal forcing as the strength of steady-state Hadley circulations. From the diagnostics presented in their paper, however, it is difficult to judge how strongly the circulations deviate from the angularmomentum-conserving limit in the equinox regime and how they transition to the nearly angular momentumconserving solstice regime.

As in the eddy-permitting simulations, the transitions in the time-dependent axisymmetric simulation are accompanied by changes in circulations fields that occur more rapidly than can be explained by the steady-state response to the thermal forcing. The seasonal cycle of the same circulation fields, shown in Fig. 6 for the steady circulations, is shown in Fig. 10 for the time-dependent circulation. At the transition of the Hadley cell from the linear to the nonlinear regime, the streamfunction extremum shifts from the winter hemisphere to a position close to the equator on a time scale shorter than the time scale of variations in the thermal forcing. With it, the inner boundary of the region of the lower-level upward mass flux moves rapidly into the summer hemisphere (Fig. 10b). The strength of the lower-level upward mass flux also increases rapidly, with maximum values increasing from $\sim 3 \times 10^{4}$ to $\sim 8 \times 10^{4} \mathrm{~kg} \mathrm{~m}^{-1} \mathrm{~s}^{-1}$ at the transition. After the transition, the lower-level upward mass flux increases more weakly, reaching $\sim 12 \times 10^{4} \mathrm{~kg} \mathrm{~m}^{-1} \mathrm{~s}^{-1}$ around the summer solstice (Fig. 10a). The regime transition is accompanied by relatively rapid changes in local

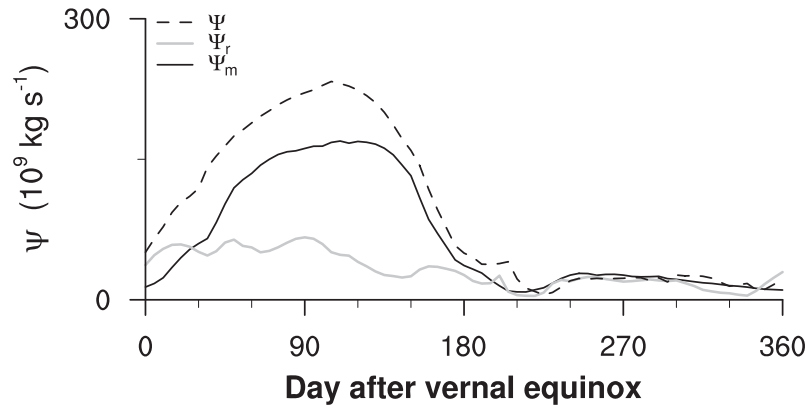

FIG. 9. Seasonal cycle of the strength of the southern Hadley cell (dashed black line) in axisymmetric simulation and $\Psi_{m}$ (black) and $\Psi_{r}$ (gray) solid lines at the center of the cell. 


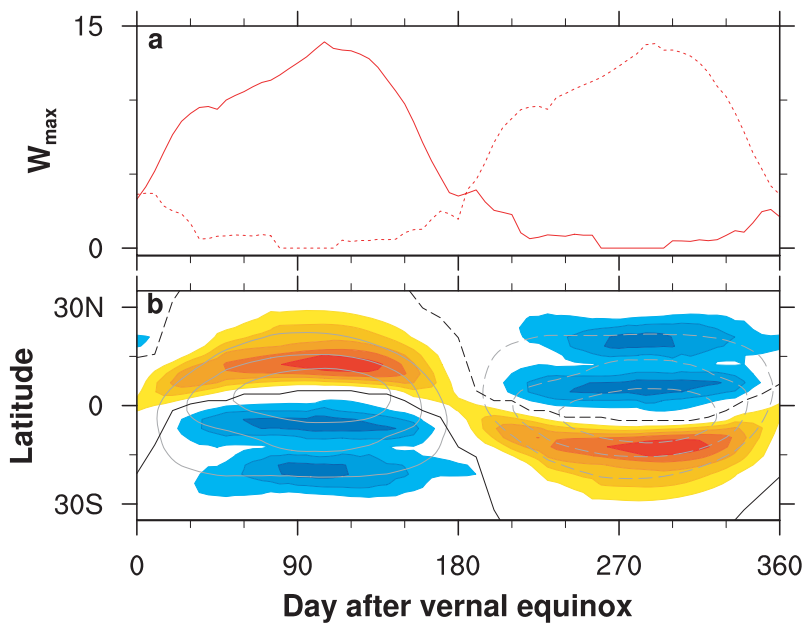

FIG. 10. Seasonal cycle of circulation fields in axisymmetric simulation. (a) Maximum value of lower-level upward mass flux $\left(10^{4} \mathrm{~kg} \mathrm{~m}^{-1} \mathrm{~s}^{-1}\right)$ in the southern Hadley cell (solid) and northern Hadley cell (dashed). (b) Quantities and contour intervals are as in Figs. $6 \mathrm{c}, \mathrm{d}$ except that the contour interval of the lower-level upward mass flux is $2 \times 10^{4} \mathrm{~kg} \mathrm{~m}^{-1} \mathrm{~s}^{-1}$.

circulation fields in the subtropics, such as an increase in the lower-level upward mass flux and a shift in the lowerlevel zonal wind from easterly to westerly (Fig. 11). Note that no reversal in the lower-level zonal wind at subtropical latitudes would occur if the circulation were simply progressing through a succession of quasi-steady states (cf. Fig. 7). These changes are qualitatively similar to those seen in the eddy-permitting simulations. As discussed below, some quantitative differences, such as the timing of the transitions and amplitude and latitudinal spread of the lower-level convergence zone, can be ascribed to the use of a longer convective time scale and nonzero vertical diffusivities in the axisymmetric simulations.

The regime transitions in the seasonal cycle of the axisymmetric Hadley cells resemble the regime transitions in the eddy-permitting simulations in SB08 in that they mark similar shifts from a linear equinox regime to a nearly angular-momentum-conserving solstice regime. The same nonlinear mean-flow feedbacks that act in the eddy-permitting simulations, and are discussed in SB08, also act at the regime transitions of the axisymmetric circulations. When the cross-equatorial Hadley cell transitions from the linear regime to the nearly angularmomentum-conserving regime, the structure of the upperlevel zonal winds is dictated by the constraint of angular momentum conservation, at least in the summer hemisphere where the influence of viscosity is negligible. This leads to the development of upper-level easterlies in the tropics, with a minimum at the equator, and, by thermal wind balance, to a negative gradient in the lower-level

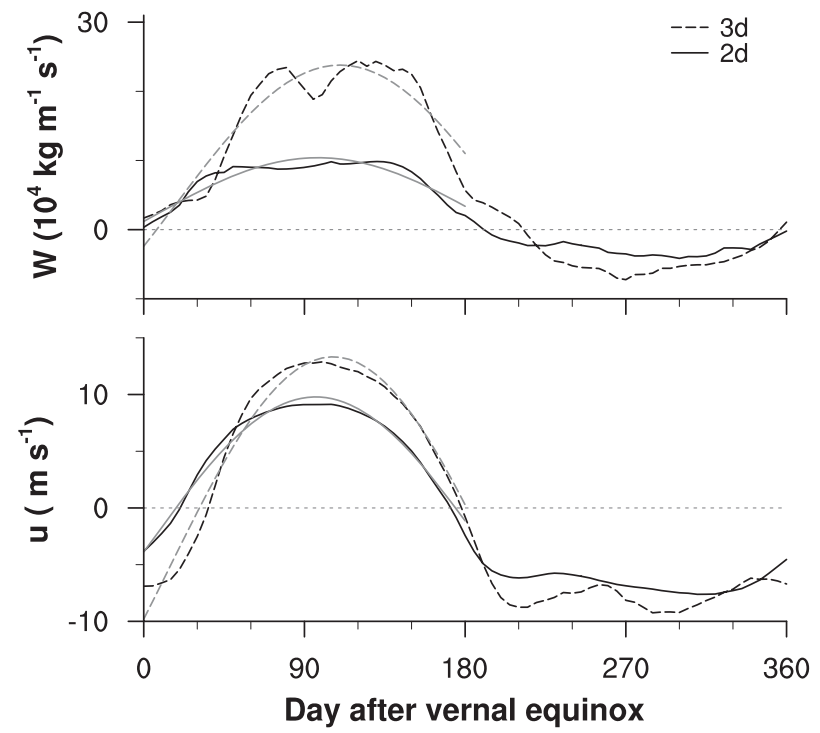

FIG. 11. Seasonal cycle of lower-level mean fields at $13^{\circ}$ latitude. (top) Upward mass flux $W$ and (bottom) zonal winds from axisymmetric (solid) and eddy-permitting (dashed) simulations. The thin gray lines show least squares sinusoidal fits to the axisymmetric (solid) and eddy-permitting (dashed) fields in the first half of the year.

potential temperature from an equatorial minimum to a subtropical maximum (cf. Lindzen and Hou 1988). The poleward advection of relatively cold air up the lowerlevel temperature gradient in the lower branch of the cross-equatorial Hadley cell pushes the subtropical temperature maximum farther poleward into the summer hemisphere. The poleward cell boundary moves with the temperature maximum (Privé and Plumb 2007a,b), which in turn leads to strengthening of the cell and strengthening and expansion into the winter hemisphere of the upper-level easterlies (rows 3 and 4 of Fig. 8). See SB08 for a more detailed discussion of this mechanism.

In the eddy-permitting simulations in SB08, another feedback-between the upper-level zonal winds and large-scale eddies of midlatitude origin-acts at the regime transitions. This feedback is absent in the axisymmetric simulations. As the Hadley cell acquires significant cross-equatorial flow at the transition to the nearly angularmomentum-conserving regime, upper-level easterlies develop in the tropics both in the summer and winter hemisphere. In the eddy-permitting simulations, the development of strong upper-level easterlies prevents the energy-containing midlatitude eddies in the winter hemisphere, which are primarily confined to regions of upper-level westerlies (Charney 1969; Webster and Holton 1982), from reaching the center and ascending branch of the cross-equatorial cell. This allows the cell to approach the angular-momentum-conserving limit more closely, which in turn leads to strengthening of the cell 
and strengthening and extension into the winter hemisphere of the upper-level easterlies, further shielding the cross-equatorial cell from the midlatitude eddies. The fact that in the time-dependent axisymmetric simulations, in which the eddy-mean flow feedback is absent, the Hadley cell still undergoes transitions between the linear and the nonlinear regime suggests that such feedback is not necessary for these transitions to occur. But, the feedback sharpens the transitions, when it acts, because circulation fields such as streamfunction strength and lower-level winds change more rapidly in the eddypermitting simulations than in the axisymmetric simulations (see Fig. 11 and compare Fig. 10 with Fig. 6 in SB08).

One other aspect in which the axisymmetric and eddypermitting simulations differ is that in the nonlinear, nearly angular-momentum-conserving regime, the lowerlevel mass flux in the axisymmetric simulations is weaker and occurs in a wider convergence zone (cf. Fig. 10 with Fig. 6 in SB08). Because the dynamics of the lower-level convergence zone is controlled in both the axisymmetric and eddy-permitting simulations by the same boundarylayer momentum balance discussed in SB08, these differences cannot be ascribed to the presence or absence of the large-scale eddies. Rather, they result from the use of a long convective relaxation time ( $\tau=1$ day) and finite vertical diffusivities $\left(\nu=5 \mathrm{~m}^{2} \mathrm{~s}^{-1}\right)$ in the axisymmetric simulations. To assess the impact of the convective time scale and vertical diffusion on the simulated circulations, we have performed eddy-permitting timedependent simulations with the longer convective time scale and/or with the vertical diffusivities of the axisymmetric simulations. In all simulations with either or both of the convective time scale and the vertical diffusivities changed, the Hadley circulations continue to undergo rapid regime transitions between the eddy-dominated and the nearly angular-momentum-conserving regime, albeit with differences in the timing of the transitions within the seasonal cycle. Both the longer convective time scale and the finite vertical diffusion influence the boundary layer dynamics, but it is the longer convective time scale that primarily causes the lower-level convergence to attain smaller values and to spread over a wider latitude band than seen in the SB08 simulation. This likely occurs because, if the convective time scale is long and hence the convective heating weak, the upward mass flux required to satisfy large-scale constraints cannot be provided in a narrow convergence zone but must spread over a wider zone. In the eddy-permitting simulation with the same convective time scale and the same vertical diffusivities as in the axisymmetric simulations, the rapid transitions of the Hadley cell between the equinox and solstice regimes are accompanied by changes in the strength and location of the lower-level convergence zone that resemble those in the axisymmetric simulations discussed here. However, it remains true that the transitions in the eddy-permitting simulations are accompanied by more rapid changes in circulation fields, such as streamfunction strength and lower-level winds, pointing to the importance of eddymean flow feedbacks in sharpening the transitions.

\section{Conclusions}

To explore if and to what extent the rapid regime transitions of the Hadley cells in the eddy-permitting simulations in SB08 and Bordoni and Schneider (2008) can still occur when large-scale eddies are suppressed, we have performed steady-state and time-dependent axisymmetric simulations. Although finite vertical diffusion of momentum and dry static energy needs to be used to achieve approximately steady states, the Hadley cells in the axisymmetric steady-state simulations generally approach the nearly inviscid limit. As the latitude of maximum radiative-equilibrium temperature is progressively displaced off the equator, they do not undergo regime transitions. The marked shifts in circulation fields that occur at the transitions from the eddy-dominated regime to the nearly angular-momentum-conserving regime in the eddy-permitting steady-state simulations do not occur in the axisymmetric steady-state simulations. As a consequence, in the axisymmetric steadystate simulations, the strength of the cross-equatorial Hadley cell, the location and intensity of the main convergence zone, and the upper- and lower-level winds in the summer subtropics do not change as rapidly as in the corresponding eddy-permitting simulations.

If the axisymmetric steady states were attained in simulations with seasonally varying thermal forcing, the tropical Hadley cells in the absence of large-scale eddies would not undergo rapid transitions between the equinox and the solstice regime, and circulation fields would change smoothly over the course of the seasonal cycle. However, in axisymmetric simulations with finite and constant vertical diffusivities, the nearly inviscid limit is only approached in the solstitial cross-equatorial Hadley cells. The time-dependent Hadley circulation undergoes transitions between an approximately linear and transient equinox regime to a nonlinear, nearly angularmomentum-conserving solstice regime. These transitions resemble the regime transitions in the eddy-permitting simulations in SB08 but differ from the latter in that time dependence of the zonal wind in the equinox regime plays a greater role in the axisymmetric simulations. Because the same nonlinear mean flow feedbacks that act in the eddy-permitting simulations are also acting at the transitions of the axisymmetric Hadley circulations, rapid 
circulation changes accompany the transitions. The absence of the additional eddy-mean flow feedback discussed in SB08 appears to explain why the circulation changes, although rapid compared to the time scale of changes in the thermal forcing, appear less sharp than those occurring in the eddy-permitting simulations.

The results from both steady-state and time-dependent axisymmetric simulations highlight how nearly inviscid theories cannot be simultaneously relevant for the equinox and cross-equatorial winter Hadley cells and cannot account for important features of the seasonal cycle. For example, the rapid regime transitions of the Hadley circulation associated with the existence and rapidity of monsoon transitions in the earth's atmosphere do not occur when Hadley cells approach the nearly inviscid limit throughout the year. This points to the importance of understanding large-scale extratropical eddies and their interaction with the tropical circulation to fully understand the dynamics of Hadley and monsoon circulations.

Acknowledgments. SB was supported by NCAR's Advanced Study Program. We are grateful for support by a David and Lucile Packard Fellowship. The simulations were carried out on Caltech's Geological and Planetary Sciences Dell cluster. We thank Rich Rotunno, Mitch Moncrieff, Rodrigo Caballero, and an anonymous reviewer for thoughtful comments on the manuscript.

\section{REFERENCES}

Boos, W. R., and K. A. Emanuel, 2008a: Wind-evaporation feedback and abrupt seasonal transitions of weak, axisymmetric Hadley circulations. J. Atmos. Sci., 65, 2194-2214.

$\longrightarrow$, and _ 2008b: Wind-evaporation feedback and the axisymmetric transition to angular momentum-conserving Hadley flow. J. Atmos. Sci., 65, 3758-3778.

Bordoni, S., and T. Schneider, 2008: Monsoons as eddy-mediated regime transitions of the tropical overturning circulation. Nature Geoscience, 1, 515-519, doi:10.1038/ngeo248.

Caballero, R., R. T. Pierrehumbert, and J. L. Mitchell, 2008: Axisymmetric, nearly-inviscid circulations in non-condensing radiative-convective atmospheres. Quart. J. Roy. Meteor. Soc., 134, 1269-1285.

Charney, J. G., 1969: A further note on large-scale motions in the tropics. J. Atmos. Sci., 26, 182-185.

Emanuel, K. A., 1995: On thermally direct circulations in moist atmospheres. J. Atmos. Sci., 52, 1529-1534.
Fang, M., and K. K. Tung, 1999: Time-dependent nonlinear Hadley circulation. J. Atmos. Sci., 56, 1797-1807.

Gadgil, S., 2003: The Indian monsoon and its variability. Annu. Rev. Earth Planet. Sci., 31, 429-467.

Held, I. M., and A. Y. Hou, 1980: Nonlinear axially symmetric circulations in a nearly inviscid atmosphere. J. Atmos. Sci., 37, 515-533.

Lindzen, R. S., and A. Y. Hou, 1988: Hadley circulations for zonally averaged heating centered off the equator. J. Atmos. Sci., 45, 2416-2427.

Newell, R. E., J. Kidson, D. G. Vincent, and G. J. Boer, 1972: The General Circulation of the Tropical Atmosphere and Interactions with Extratropical Latitudes. Vol. I. MIT Press, 258 pp.

Plumb, R. A., and A. Y. Hou, 1992: The response of a zonally symmetric atmosphere to subtropical thermal forcing: Threshold behavior. J. Atmos. Sci., 49, 1790-1799.

Privé, N. C., and R. A. Plumb, 2007a: Monsoon dynamics with interactive forcing. Part I: Axisymmetric studies. J. Atmos. Sci., 64, 1417-1430.

$\longrightarrow$, and - 2007b: Monsoon dynamics with interactive forcing. Part II: Impact of eddies and asymmetric geometries. J. Atmos. Sci., 64, 1431-1442.

Satoh, M., 1994: Hadley circulations in radiative-convective equilibrium in an axially symmetric atmosphere. J. Atmos. Sci., 51, 1947-1968.

Schneider, E. K., 1977: Axially symmetric steady-state models of the basic state for instability and climate studies. Part II: Nonlinear calculations. J. Atmos. Sci., 34, 280-296.

Schneider, T., 2006: The general circulation of the atmosphere. Annu. Rev. Earth Planet. Sci., 34, 655-688.

— the seasonal cycle of a Hadley circulation and implications for monsoon dynamics. J. Atmos. Sci., 65, 915-934.

Sobel, A. H., and T. Schneider, 2009: Single-layer axisymmetric model for a Hadley circulation with parameterized eddy momentum forcing. J. Adv. Model Earth Syst., 1, doi:10.3894/ JAMES.2009.1.10.

Walker, C. C., and T. Schneider, 2005: Response of idealized Hadley circulations to seasonally varying heating. Geophys. Res. Lett., 32, L06813, doi:10.1029/2004GL022304.

— Simulations with an idealized GCM. J. Atmos. Sci., 63, 33333350 .

Webster, P. J., 1987: The elementary monsoon. Monsoons, J. S. Fein and P. L. Stephens, Eds., John Wiley \& Sons, 3-32.

_ , and J. R. Holton, 1982: Cross-equatorial response to middlelatitude forcing in a zonally varying basic state. J. Atmos. Sci., 39, 722-733.

Zheng, X. Y., 1998: The response of a moist zonally symmetric atmosphere to subtropical surface temperature perturbation. Quart. J. Roy. Meteor. Soc., 124, 1209-1226. 